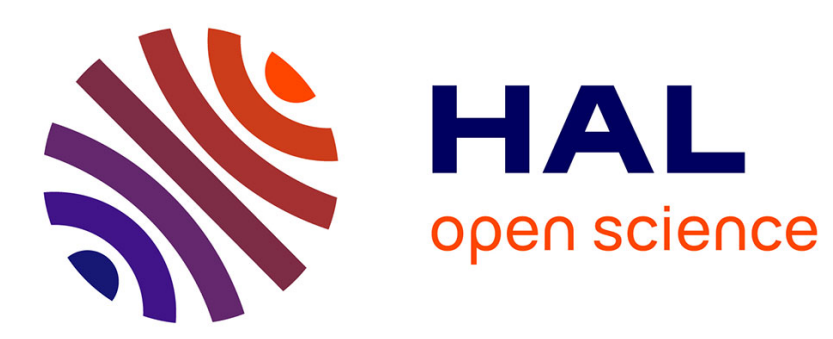

\title{
Élargissement et déplacement de la raie $1849 \AA$ Å absorbée par la vapeur de mercure en présence d'argon
}

\author{
C. Bousquet, N. Bras, R. Lennuier
}

\section{To cite this version:}

C. Bousquet, N. Bras, R. Lennuier. Élargissement et déplacement de la raie $1849 \AA$ absorbée par la vapeur de mercure en présence d'argon. Journal de Physique Lettres, 1975, 36 (10), pp.233-234. 10.1051/jphyslet:019750036010023300 . jpa-00231195

\section{HAL Id: jpa-00231195 https://hal.science/jpa-00231195}

Submitted on 1 Jan 1975

HAL is a multi-disciplinary open access archive for the deposit and dissemination of scientific research documents, whether they are published or not. The documents may come from teaching and research institutions in France or abroad, or from public or private research centers.
L'archive ouverte pluridisciplinaire HAL, est destinée au dépôt et à la diffusion de documents scientifiques de niveau recherche, publiés ou non, émanant des établissements d'enseignement et de recherche français ou étrangers, des laboratoires publics ou privés. 


\title{
ÉLARGISSEMENT ET DÉPLACEMENT DE LA RAIE 1849 A ABSORBÉE PAR LA VAPEUR DE MERCURE EN PRÉSENCE D'ARGON
}

\author{
C. BOUSQUET, N. BRAS et R. LENNUIER \\ Département de Recherche Physique (*) Université Pierre et Marie-Curie \\ 4, place Jussieu, Tour 22, 75230 Paris Cedex 05, France
}

(Reçu le 16 mai 1975, accepté le 19 juin 1975)

\begin{abstract}
Résumé. - Description d'un dispositif de balayage magnétique permettant d'analyser, à l'intérieur d'un domaine spectral d'une largeur de $10 \mathrm{GHz}$, le profil de la raie $1849 \AA \AA$ du mercure. L'élargissement et le déplacement ont été mesurés lorsque de l'argon sous faible pression est ajouté à la vapeur de mercure monobare 198. La section efficace correspondante a été trouvée égale à (314 \pm 34$) 10^{-16} \mathrm{~cm}^{2}$ à la température de $287 \mathrm{~K}$.
\end{abstract}

\begin{abstract}
In this paper we describe a magnetic scanning apparatus with a spectral range of $10 \mathrm{GHz}$ used to analyse the profile of the resonance radiation $\mathrm{Hg} 1849 \AA$. The width and the shift of this line have been measured when argon is introduced in to an absorption cell containing ${ }^{198} \mathrm{Hg}$. The cross-section for the broadening of the line is $(314 \pm 34) 10^{-16} \mathrm{~cm}^{2}$ at a temperature of $287 \mathrm{~K}$.
\end{abstract}

1. Introduction. - L'étude des déformations du profil spectral des raies sous l'effet des collisions résonnantes et non résonnantes subies par l'atome de mercure avait principalement concerné jusqu'ici dans notre laboratoire la transition $6{ }^{1} \mathrm{~S}_{0} \rightarrow 6{ }^{3} \mathrm{P}_{1}$ (raie $2537 \AA$ ) [1, 2]. Il est souhaitable d'obtenir des informations analogues pour la transition $6{ }^{1} \mathrm{~S}_{0} \rightarrow 6{ }^{1} \mathrm{P}_{1}$, mais la raie correspondante (1 $849 \AA$ \&) est d'une étude expérimentale plus difficile. En particulier, l'exploration de la partie centrale du profil, où se manifeste l'effet des collisions satisfaisant aux conditions d'impact, exige une analyse spectrale à très haute résolution. La transposition à ce domaine spectral de l'analyse par balayage magnétique utilisée pour la raie $2537 \AA$ avait été réalisée par E. Leboucher et avait donné quelques résultats [3]. Mais la raie $1849 \AA$, extrêmement auto-absorbable, présente une sensibilité aux conditions de fonctionnement des sources telle que son profil se déforme lorsque varie l'induction magnétique appliquée à celles-ci au cours du balayage. Il a donc fallu renoncer au dispositif de balayage magnétique qui plaçait la source dans le champ magnétique variable. Il pouvait être tentant de placer dans celui-ci la cellule de mercure monobare additionné ou non de perturbateurs étrangers dont il s'agit de déterminer le profil d'absorption sur $1849 \AA$. Mais nous voulons pouvoir interpréter les résultats en tenant compte de l'anisotropie du potentiel d'in-

(*) Laboratoire associé au C.N.R.S. n $^{\circ} 71$ teraction atome de mercure $6{ }^{1} \mathrm{P}_{1}$-atome perturbateur; aussi avons nous évité d'introduire une direction privilégiée dans la région où se trouvent les atomes étudiés. Nous avons donc adopté un dispositif de balayage magnétique plus compliqué, laissant hors du champ magnétique à la fois la source et la cellule contenant les gaz à étudier.

2. Dispositif expérimental. - L'absorption du rayonnement de longueur d'onde $1850 \AA$ par l'oxygène atmosphérique est évitée en plaçant toutes les parties du montage optique dans des enceintes étanches où un bon vide primaire est maintenu.

La source, un tube assez fin (diamètre intérieur $2 \mathrm{~mm}$ ) rempli de mercure monobare 198, excité en H.F. (500 MHz) fonctionne dans des conditions telles (refroidissement à $-20^{\circ} \mathrm{C}$ ) qu'elle émet sur $1849 \AA$ un profil $I(v)$ assez plat sur une largeur de l'ordre de $10 \mathrm{GHz}$. Ce profil I(v) sera invariable au cours de l'expérience. Une cellule de référence $C_{0}$ (épaisseur $l_{0}=0,1 \mathrm{~mm}$ ) maintenue à température fixe (généralement à $287 \mathrm{~K}$ ) contient du mercure monobare 198; une cellule $C_{0}^{\prime}$ identique, placée dans la même enceinte, contient du mercure monobare 198 additionné d'une certaine quantité d'argon; $\mathrm{C}_{0}$ et $\mathrm{C}_{0}^{\prime}$ sont alternativement interposées sur un faisceau issu de la source. Soient $k_{0}(v)$ et $k_{0}^{\prime}(v)$ leurs coefficients d'absorption respectifs; les profils d'absorption qui en résultent ont des largeurs inférieures à celle du profil $I(v)(\mathrm{du}$ moins, pour $\mathrm{C}_{0}^{\prime}$, à condition de limiter la pression d'argon à des valeurs inférieures à 200 torrs). Le 
profil résultant de leur interposition sur le faisceau est donc, soit $I(v) \mathrm{e}^{-k_{0}(v) l_{0}}$, soit $I(v) \mathrm{e}^{-k_{0}^{\prime}(v) l_{0}}$. Ce sont ces profils qui vont être analysés par balayage magnétique.

A cette fin une cellule $C$ d'épaisseur $l=0,2 \mathrm{~mm}$ contenant l'isotope $202 \mathrm{du}$ mercure est placée dans l'entrefer d'un électroaimant dont l'induction peut varier de 0 à $12000 \mathrm{G}$. La pression du mercure y est contrôlée par la température du queusot, mesurée avec précision (point froid autour de $5^{\circ} \mathrm{C}$ ). Entre la source et les cellules $\mathrm{C}_{0}$ et $\mathrm{C}_{0}^{\prime}$ le faisceau chemine suivant l'axe de l'électroaimant et traverse la cellule $\mathrm{C}$. Un prisme de quartz associé à un jeu convenable de lentilles et de fentes, assure l'isolement de la raie $1849 \AA \AA$ (on a tout particulièrement veillé à ce que la radiation $2537 \AA$ soit parfaitement éliminée).

Aux faibles valeurs de l'induction $B$ la vapeur de mercure de $C$ n'absorbe pas les fréquences du profil $I(v)$. Pour $B=6500 \mathrm{G}$ environ, le profil d'absorption de l'une des composantes Zeeman de la vapeur de mercure 202 est sensiblement centré sur le profil $I(v)$. Le balayage en champ autour de cette valeur promène donc un profil d'absorption assez fin, caractérisé par le coefficient d'absorption $k\{v-v(B)\}$ où $v(B)$ est la fréquence sur laquelle l'effet Zeeman amène une des deux composantes circulaires issues de la fréquence $v_{0}(202)$. Enfin une lame séparatrice permet de recevoir sur deux photomultiplicateurs, soit un faisceau n'ayant traversé que $C$, soit un faisceau ayant traversé $C$ et $C_{0}\left(\right.$ ou $\left.C_{0}^{\prime}\right)$. On déduit facilement des signaux reçus les grandeurs suivantes qui sont des produits de convolution :

$$
y_{1}\{v(B)\}=\int_{-\infty}^{+\infty} \frac{I(v)}{\int_{-\infty}^{+\infty} I(v) \mathrm{d} v}\left\{1-\mathrm{e}^{-k\{v-v(B)\} l}\right\} \mathrm{d} v
$$

$$
\begin{array}{r}
y_{2}\{v(B)\}=\int_{-\infty}^{+\infty} \frac{I(v) \mathrm{e}^{-k_{0}(v) l_{0}}}{\int_{-\infty}^{+\infty} I(v) \mathrm{e}^{-k_{0}(v) l_{0}} \mathrm{~d} v} \times \\
y_{2}^{\prime}\{v(B)\}=\int_{-\infty}^{+\infty} \frac{I(v) \mathrm{e}^{-k_{0}^{\prime}(v) l_{0}}}{\int_{-\infty}^{+\infty} I(v) \mathrm{e}^{-k_{0}^{\prime}(v) l_{0}} \mathrm{~d} v} \times \\
\times\left\{1-\mathrm{e}^{-k\{v-v(B)\} l}\right\} \mathrm{d} v .
\end{array}
$$

3. Exploitation des mesures. - Nous nous sommes assurés que, dans nos conditions d'expérience, les interactions mercure-mercure sont négligeables dans tous les cas où doit être calculé un coefficient d'ab- sorption. On peut prévoir théoriquement $[4,5]$ la valeur du rapport entre l'élargissement d'une raie $\mathrm{S}_{0}-\mathrm{P}_{1}$ par interaction résonnante et la largeur naturelle; ces prévisions théoriques sont bien vérifiées par l'expérience dans le cas de la transition $6{ }^{1} \mathrm{~S}_{0}-6{ }^{3} \mathrm{P}_{1}$ du mercure [2]; elles conduisent dans le cas de la transition $6{ }^{1} \mathrm{~S}_{0}-6{ }^{1} \mathrm{P}_{1}$ du mercure à un rapport inférieur à 0,05 dans les conditions de pression de nos expériences.

On sait alors calculer a priori, compte tenu de ces conditions expérimentales, les coefficients d'absorption $k_{0}(v)$ et $k\{v-v(B)\}$. Les profils inconnus sont ceux de $I(v)$ et $k_{0}^{\prime}(v)$.

Le profil $I(v)$ s'obtient par déconvolution de (1) utilisant les transformées de Fourier; elle s'effectue sur ordinateur. La convolution (1) effectuée à partir de ce résultat doit redonner $y_{1}\{v(B)\}$, ce qui permet un premier contrôle de la fonction $I(v)$ retenue. Un second contrôle est fourni par le calcul de $y_{2}\{v(B)\}$ par la convolution (2). Pour obtenir le profil de perturbation par collision qui intervient dans $k_{0}^{\prime}(v)$ nous avons admis que, les conditions d'impact étant réalisées, celui-ci est une lorentzienne caractérisée par sa largeur à mi-hauteur $\delta v_{\mathrm{L}}$ et son déplacement $\delta v_{\mathrm{D}}$ par rapport à $v_{0}(198)$. (La référence à cette fréquence est réalisée grâce à $\mathrm{C}_{0}$ et à $\left.y_{2}\{v(B)\}\right)$. On calcule alors sur ordinateur les produits de convolution définis par (3) et on ajuste les deux paramètres $\delta v_{\mathrm{L}}$ et $\delta v_{\mathrm{D}}$ de manière à retrouver la donnée expérimentale $y_{2}^{\prime}\{v(B)\}$. Les opérations sont longues mais conduisent à des résultats cohérents dans la limite d'une précision de l'ordre de $10 \%$.

4. Résultats. - Les mesures effectuées jusqu'ici concernent la perturbation du mercure par l'argon; les pressions réalisées pour ce gaz ont été $38,80,118$ et 157 torrs. Elargissement et déplacement ont alors été trouvés proportionnels à la pression du perturbateur, avec les valeurs :

élargissement : $\delta v_{\mathrm{L}} / p=(10,8 \pm 1,2) \mathrm{GHz} /$ Atm. soit (360 \pm 40$) \mathrm{mK} /$ Atm. ;

déplacement : $\delta v_{\mathrm{D}} / p=(-3,4 \pm 0,5) \mathrm{GHz} / \mathrm{Atm}$. soit $(-112 \pm 15) \mathrm{mK} /$ Atm.

Nous pouvons calculer une section efficace $\sigma_{\mathrm{opt}}$ pour la relaxation de la cohérence optique,

$$
\sigma_{\mathrm{opt}}=1 / N \bar{v} \tau
$$

où $\tau=1 / \pi \delta v_{\mathrm{L}}$ ( $N$ nombre d'atomes perturbateurs par unité de volume, $\bar{v}$ vitesse relative moyenne des atomes du couple $\mathrm{Hg}-\mathrm{Ar}$ ). On obtient, à $T=285 \mathrm{~K}$,

$$
\sigma_{\mathrm{opt}}=(314 \pm 34) 10^{-16} \mathrm{~cm}^{2} \text {. }
$$

\section{Bibliographie}

[1] Butaux, J., Thèse Paris, 1972.

Butaux, J., Schuller, F., Lennuier, R., J. Physique 33 (1972) 635.

[2] Perrin, D., Thèse Paris, 1974.

Perrin, D., Lennuier, R., J. Physique 36 (1975) 357.
[3] Leboucher, E., Bousquet, C., Bras, N., Nouv. Revue Opt. Appl. 5 (1974) 121.

[4] Omont, A., Meunier, J., Phys. Rev. 169 (1968) 92.

[5] Berman, P. R., Lamb, W. E., Phys. Rev. 187 (1969) 221. 